\title{
Application of the grazing angle polarized neutron reflectometry to study the magnetism in thin films and stratified media
}

\author{
M. MAAZA
}

Laboratoire Leon Brillouin, Commissariat à l'Energie Atomique, Centre National de la Recherche Scientifique, Bat 563, Centre d'Etudes de Saclay, 91191 Gif-sur-Yvette, France

\begin{abstract}
RESUME
Du point de vue optique et compte tenu de l'interaction des neutrons froids avec les électrons non-appariés, les matériaux magnétiques possèdent un indice de réfraction dépendant de l'état de spin des neutrons $\mathrm{n}^{+}$[spin Up] and $\mathrm{n}^{-}$[spin Down]. Les milieux magnétiques tels que $\mathrm{Fe}$, Co et Ni reagissent comme les cristaux biréfringents uniaxiaux en optique ordinaire. $n^{+}$et $n^{-}$sont l'équivalent des indices ordinaire et extraordinaire respectivement. La réflexion spéculaire des neutrons polarisés qui est due à la discontinuité de l'induction magnétique à la surface du matériau ferromagnétique est un outil trés sensible au magnétisme de surface et d'interface. Dans un premier temps, nous donnerons les relations de base décrivant l'optique de neutrons polarisés. Dans une seconde étape, des exemples récents sur le magnétisme de surface et d'interface dans les films minces et multicouches tels que le couplage magnétique et la mesure de profondeur de pénétration dans les supraconducteurs, seront donnés afin d'illustrer la puissance de cette technique.

\section{ABSTRACT}

From optical point of view and due to the magnetic interaction of the cold neutrons with the unpaired electron shell, magnetic materials have a neutron spin-dependent refractive index $\mathrm{n}^{+}$[spin up] and $\mathrm{n}^{-}$[spin down]. Magnetic media such as $\mathrm{Fe}, \mathrm{Co}$ and $\mathrm{Ni}$ react like birefringent uniaxial crystals in ordinary optics. $n^{+}$and $n^{-}$are the equivalent of the ordinary and extraordinary refractive indices. The specular reflection of spin polarized neutrons which is due to the discontinuity of the magnetic induction at the surface of the ferromagnet is a sensitive probe of surface and interface magnetism. We shall first give the background of the art of polarized neutron optics. Secondly, some recent examples from surface and interface magnetism will be given to illustrate the power of this technique such as the magnetic coupling in thin films and multilayers and flux penetration in superconductors.
\end{abstract}

\section{INTRODUCTION}

There are several powerful methods to study the surface and interface magnetism on macroscopic and microscopic scale to understand the magnetic behaviour and the application properties of the materials [1-3]. The magnetometry is used to measure mean value of the magnetic moments whilst the magnetic surface anisotropy is measured by ferromagnetic resonance, Brillouin light-scattering and torsion oscillation magnetometry. The spin polarized secondary electrons which allow to analyze the spin-polarized electronic surface structure is restricted to external surfaces. This is due to the small depth penetration of electrons. Mössbauer spectroscopy and nuclear magnetic resonance follow the variation of the local structure of magnetic hyperfine fields. Some optical techniques are also used to study the surface and interface magnetism such as surface magneto-optics Kerr effect and $\mathrm{X}$-ray circular dichroism. 
According to the weak absorption of the cold neutrons and so their strong penetration depth [of the order of $\mu \mathrm{m}$ ], it is possible to study both surface and interface magnetism in both thick layers and stratified media. Moreover, in the previous magnetic techniques, it is very difficult to measure the absolute value of the local moment [4]. As it was demonstrated by Felcher [5] and Bland et al [6], it is possible to do absolute measurements with the grazing angle polarized neutron reflectometry.

\section{THEORETICAL BACKGROUND}

The interactions of cold neutrons with a magnetic material are nuclear and magnetic, characterized by coherent nuclear and magnetic scattering lengths $b_{n}$ and $b_{m}$ respectively [7]. $b_{n}$ can be positive or negative whilst $b_{m}$ is always positive[7] [table 1].

Table 1.: Values of the spin-dependent scattering lengths for $3 \mathrm{~d}$ electron ferromagnets $\left[\times 10^{-12} \mathrm{~cm}\right]$ at saturation and titanium.

\begin{tabular}{lllll} 
Element & $\begin{array}{l}\text { Magnetic } \\
\text { scattering } \\
\text { amplitude } \\
\mathrm{b}_{\mathrm{m}}\end{array}$ & $\begin{array}{l}\text { Nuclear } \\
\text { scattering } \\
\text { amplitude } \\
\mathrm{b}_{\mathrm{n}}\end{array}$ & $\begin{array}{l}\text { spin Up } \\
\text { scattering } \\
\text { amplitude } \\
\mathbf{b}^{+}\end{array}$ & $\begin{array}{l}\text { spin Down } \\
\text { scattering } \\
\text { amplitude } \\
\mathbf{b}^{-}\end{array}$ \\
\hdashline $\mathrm{Ni}$ & 0.162 & 1.03 & 1.19 & 0.87 \\
$\mathrm{Co}$ & 0.464 & 0.250 & 0.714 & -0.214 \\
$\mathrm{Fe}$ & 0.589 & 0.954 & 1.552 & 0.356 \\
$\mathrm{Ti}$ & 0 & -0.34 & -0.34 & -0.34 \\
\hline
\end{tabular}

From the optical point of view, the interaction between cold neutrons entering a non-absorbant magnetic material is described by a refractive index given by [8] :

$\mathrm{n}_{ \pm}(\mathrm{z})=1-\lambda^{2} \mathcal{N}\left[b_{n}(z) \pm b_{m}(z)\right] / 2 \pi$

$b_{m}(z)=2 \pi \mu_{n} m_{n} H(z) / h^{2} \mathfrak{N}$

where the $z$ axis is perpendicular to the reflecting surface, $m_{n}$ and $\mu_{n}$ are the mass and the magnetic moment of the neutron of de Broglie associated wavelength $\lambda, \lambda b_{n}(z)$ and $\mathrm{Ob}_{\mathrm{m}}(\mathrm{z})$ are the mean bound coherent nuclear and magnetic scattering length densities in the material respectively. The choice of sign is determined by whether the nuclear moment is aligned parallel [ +$]$ or anti-parallel [-] to the applied field $H(z)$. Equation (1) shows that the refractive index is highly dispersive and and in magnetized materials is neutron-spin dependent. This property is exploited in all optical neutron magnetic studies and applications [9-11]. Since $b_{n}(z)$ may be of either sign, $n \pm(z)$ may be smaller or greater than unity. In the latter case, total mirror reflection of neutrons may occur at glancing angles less than a critical angle $\theta_{c \pm}(z)$ given closely by [12]:

$\left[\theta_{c} \pm\right]^{2} \approx \lambda^{2} \mathcal{N}\left[b_{n}(z) \pm b_{m}(z)\right] / \pi$ 


\section{Ferromagnetic mirror}

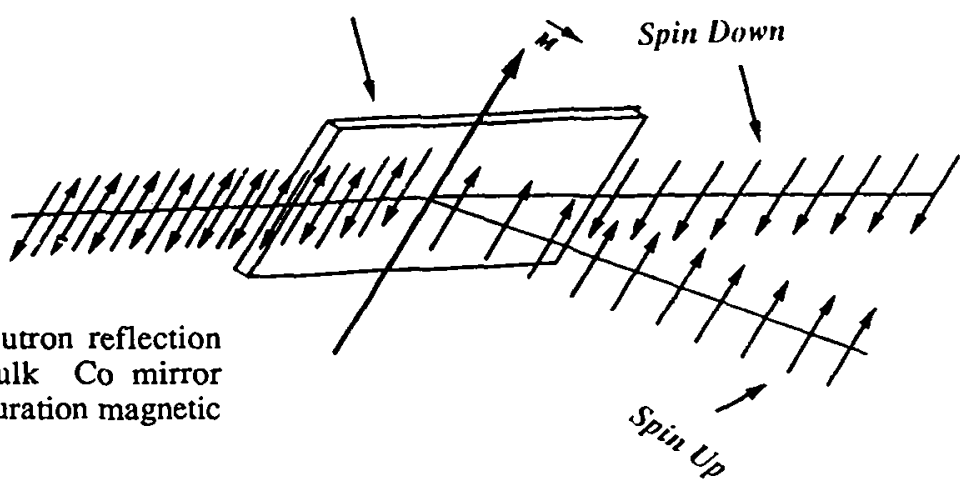

Fig 1: Neutron reflection on a magnetic bulk Co mirror device under a saturation magnetic external field $H$.

If assumed that the geometry is such that the material is magnetized in a field which is applied parallel to its surface as shown in figure 1. This figure illustrates the case of a cobalt mirror under saturation magnetic field. There is no total reflection for spin Down neutrons. In general, the spin reflectivities of a magnetic semi-infinite slab in which the magnetization is constant are given by [13]:

$\left.\left.R \pm(\theta / \lambda)=\left\{\sin \theta-\sqrt{[n \pm} \pm^{2}-\cos ^{2} \theta\right]\right\} /\left\{\sin \theta+\sqrt{[n \pm}{ }^{2}-\cos ^{2} \theta\right]\right\}$

If there is a change in the magnetic structure of the considered slab, the calculated dependence of both the reflectivities $R_{+}$and $R_{\text {. }}$ as a function of the reflection angle $\theta$ or neutron associated wavelength $\lambda$ or momentum $K=[2 \pi \sin \theta] / \lambda$ allows to determine the magnetization depth profile $\mathrm{H}(\mathrm{z})$ by subdivising the mirror material into a series of thin slices, with thicknesses $d_{j}$ and characterized by its own refractive index $n_{j \pm}$. The classical optics method is used for calculating the reflection and the transmission coefficients at each interface [14] and hence $R_{+}, R_{-}$and their ratio so called flipping ratio $F(\theta / \lambda)=$ $\left[R^{+} / R^{-}\right]$. There are two main methods to generate polarized cold neutron beam. The first method is based on the existence of two critical glancing angles for total neutron reflection from magnetized mirrors such as a cobalt or a vanadium permendur mirror [15]. The second one consists of employing multilayered polarizing mirrors constituted by a succession of magnetic and non magnetic materials [16-18] such as [Fe-Ge] supermirrors. The measurement technique can use the time of flight technique or/and $\theta$ $2 \theta$ geometry [19].

\section{ILLUSTRATIVE EXAMPLES}

\subsection{Nuclear and magnetic reflections}

To illustrate the difference between the nuclear and the magnetic reflections, a 10 bilayers of [ $\mathrm{Ni}$-Ti] superlattice deposited onto a borosilicate glass. The expected thickness of $\mathrm{Ni}$ and $\mathrm{Ti}$ layers are $\mathrm{d}_{\mathrm{Ni}}$ the $=\mathrm{d}_{\mathrm{Ti}}$ the $=400 \AA$. To investigate the overall characteristics of this periodic stack, the superlattice is first scanned on the unpolarized neutron reflectometer DESIR localized at Léon Brillouin Laboratory at $\theta_{0} \approx 2.410^{-2} \mathrm{rad}$ [19]. 
Fig 2.a.: Observed grazing angle unpolarized neutron reflectometry profiles $[\log R]$ of the $10[400 \AA \AA \mathrm{Ni}-400 \AA \mathrm{Ti}] /$ Borfron glass multilayer [ooooo] versus the momentum and the corresponding theoretical $[\cdots \cdots \cdot]$ and simulated [----] unpolarized neutron reflectivity profiles.

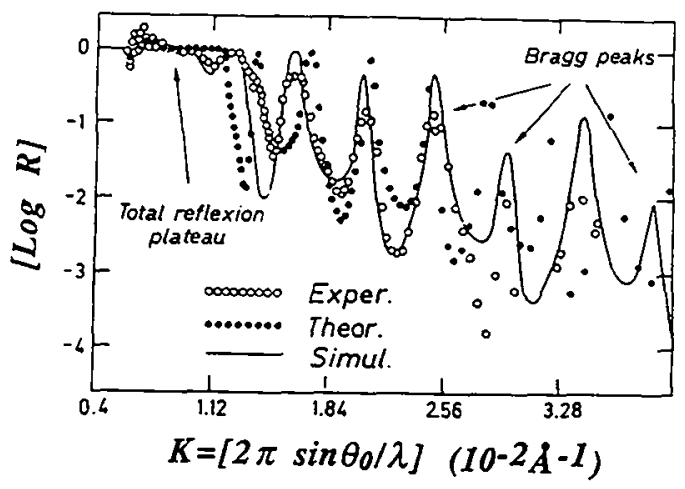

As shown in figure 2.a., many Bragg order were detected. Assuming uniform densities and sharp interfaces, the $\mathrm{Ni}$ and $\mathrm{Ti}$ layers can be considered to be nominally about $\mathrm{d}_{\mathrm{Ni}}$ sim $=\mathrm{d}_{\mathrm{Ti}}$ sim $=330 \AA$. The simulated value of the superlattice period is then $\Lambda^{\operatorname{sim}}=660$ against $\Lambda^{\text {the }}=800 \AA$ which is the expected value. This high discreapancy suggests that some diffusion has taken place during the sputtering. The simulated neutron scattering length densities are $2 \mathrm{~b}_{\mathrm{Ni}}$ sim $=+9.410^{6} \AA^{-2}$ and $\mathcal{N} \mathrm{Ti}_{\mathrm{Ti}} \mathrm{sim}=-0.5$ $10^{6} \AA-2$ respectively. $2 b_{\mathrm{Ni}}$ sim corresponds to the pure $\mathrm{Ni}$ whereas the $2 \mathrm{~N}_{\mathrm{Ti}}^{\mathrm{Ti}} \mathrm{sim}$ one is less negative than the pure $\mathrm{Ti}$. This indicates a diffusion of $\mathrm{Ni}$ into $\mathrm{Ti}$ [20] which confirms the previous hypothesis.

Fig 2.b.: Observed grazing angle polarized neutron reflectometry profiles $[\log R]$ of the $10[400 \AA \mathrm{Ni}-400 \AA \mathrm{Ti}] /$ borosilicate glass multilayer versus the momentum $\mathrm{K}=2 \pi \sin \theta_{0} / \lambda$ : Spin

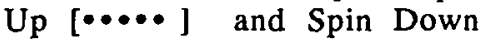
[000].

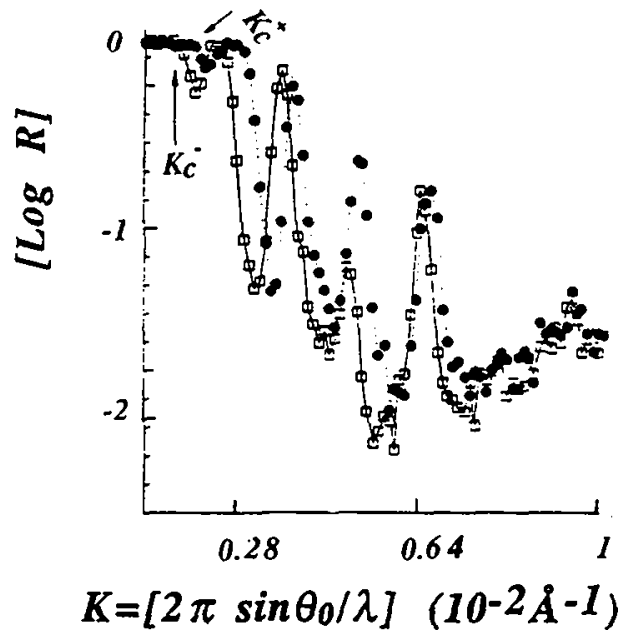

Secondly, polarized neutron measurements were carried out on the D17 polarized neutron reflectometer at Institut Laüe-Langevin [16]. The reflectivity curves are measured with a goniometer by $\theta-2 \theta$ scan with a fixed neutron wavelength $\lambda=7.2 \AA$. The $[+]$ and $[-$ ] neutron spin reflectivities are shown in figure 2.b. It should be noted that the $[+] \mathrm{Bragg}$ peaks are shifted relative to the $[-]$ ones. Moreover, the $[+]$ spin reflectivity becomes less than the $[-]$ spin at the third-order Bragg position at $K_{B}[k=3]=0.51 \quad 10^{-2} \AA^{-1}$. This is most easily explained by assuming that some interdiffusion takes place between $\mathrm{Ni}$ and 
Ti layers [21-22] which is in agreement with the previous unpolarized results. As indicated above, the critical parameters $\mathrm{K}_{\mathrm{c}}^{+}=2 \pi \sin \theta_{0} / \lambda_{\mathrm{c}}^{+}$and $\mathrm{K} \bar{c}=2 \pi \sin \theta_{0} / \lambda_{\mathrm{c}}$. are different; their average values are $0.3110^{-2} \AA^{-1}$ and $0.2710^{-2} \AA^{-1}$ respectively. This indicates that $\mathrm{Ni}$, far from the interfacial region, is magnetic, in spite the dead magnetic interfacial layer. This kind of test can be generalized to monolayers of a magnetic material.

\subsection{Superconductor thin films}

Felcher et al [5] have measured the penetration of the magnetic field $H(z)$ into the surface of a bulk superconducting niobium. The magnetic field profile as a function of penetration depth $z$ into the niobium was assumed to have the form $H(z)=\mathrm{H} \mathrm{e}^{-\mathrm{z} / \Lambda(0)}$ where $\Lambda(0)$ is a characteristic penetration depth. This superconducting penetration depth was of the order of $[410 \pm 40] \AA$. In the same material, Chernenko et al [23] have carried out experiments on a polycristalline niobium thin film at the temperature $\mathrm{T}=4.9 \mathrm{~K}$ in a field of 500 Oe on the SPN reflectometer localized at the IBR-2 reactor in Dubna [24]. The film which thickness is [2550 \pm 150$] \AA$ is sputtered on silicon substrate. The critical temperature $T_{c}=8.95 \mathrm{~K}$. To analyse the experimental data, the authors used the diamagnetic profile of a superconducting film according to London's local electrodynamics of superconductors.

Fig 3: Experimental values of the flipping ratio [*... ] and simulated calculations [--- ] considering the corrections for polarization and resolution of the reflectometer versus $[\lambda / \sin \theta]$ of a thin Nb film [2550Ả]. Dashed lines are calculated for superconducting penetration depth of $500 \AA$ found by Felcher et al [5] for bulk Niobium (From Chemenko et al [23]).

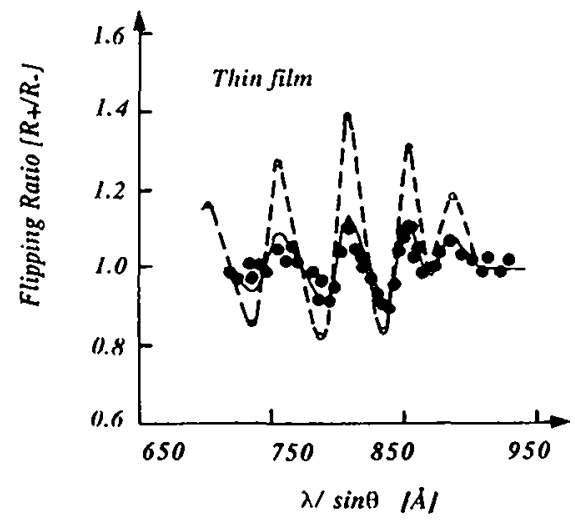

Figure 3 shows the experimental variation of the flipping ratio versus the momentum and the corresponding fit at $T=4.9 \mathrm{~K}$. From this fit, the value of the

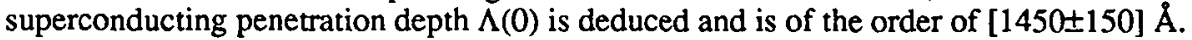
The value of superconducting penetration depth found by Felcher et al on bulk niobium $\Lambda(0)=430 \AA$, describes this new result adequately with a dead layer of a thickness of $1000 \AA$ at the substrate interface, which does no turn into a superconducting state at $\mathrm{T}=4.9 \mathrm{~K}$. The hypothesis of the dead layer of $1000 \AA$ is not appropriate for two reasons. First, the analysis of the chemical depth composition of the thin niobium film by backward scattering of accelerated helium ions has shown that there is neither oxygen nor other impurities across the whole width of the thin film. Secondly, the thin films of a thickness of $1000 \AA$ are superconducting at such a temperature: $\mathrm{T}=4.9 \mathrm{~K}$. 


\subsection{Magnetic moments in ultra-thin films and interfacial anisotropy}

Theoretical calculations of the electronic and magnetic structure of epitaxial films using the recently developed spin resolved band structure techniques $[2,25]$ demonstrate that the reduced atomic coordination, modified volume per atom and the interfaceinduced electronic states strongly affect the moment. In this way, polarized neutron reflectometry can be applied to the study of the absolute value of the magnetic moment per atom of sandwiched ultra-thin epitaxial films and magnetic layers. Figure 4 shows the observed polarization of the reflected beam at $4 \mathrm{~K}$ for a sandwich constituted by ten monolayers of fcc $\mathrm{Co}[18 \AA \AA$ ] epitaxially deposited onto a $\mathrm{Cu}(001)$ substrate overcoated with $42 \AA$ of $\mathrm{fcc} \mathrm{Cu}$. These measurements were made with the previous polarized reflectometer D17 [26]. The position of the peak maximum is very sensitive to the thickness $d_{2}$ of the

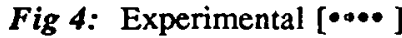
and simulated $[----]$ Spin Assymetries observed at $4 \mathrm{~K}$ for a $42 \AA \mathrm{Cu} / 18 \AA \mathrm{Co} / \mathrm{Cu}(001)$ compared to experimental flipping ratio of [aria] $50 \AA \mathrm{Cu} / 9 \AA \mathrm{Fe} / \mathrm{Rh}(001)$

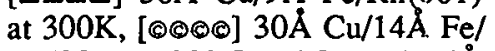
$\mathrm{Cu}(001)$ at $300 \mathrm{~K}$ and $[\Delta \Delta \Delta \Delta] 15 \AA$ $\mathrm{Au} / 12 \AA \mathrm{Fe} / 55 \AA \mathrm{Au} / \mathrm{glass}$ at $300 \mathrm{~K}$ (From Bland et al [26]).

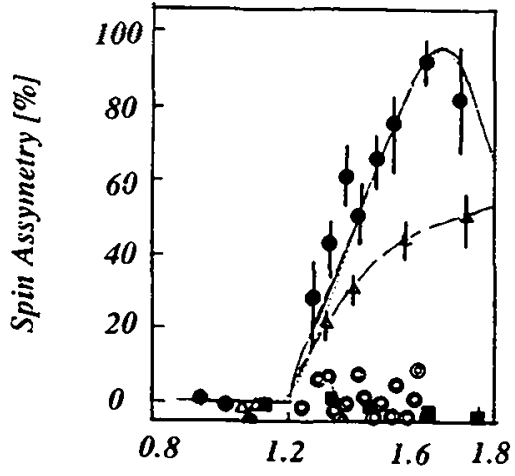

Reduced wavevector $K / K_{C}$

overlayer while its height measures the absolute value of the magnetic moment per atom. The simulation of the experimental curve gives $\mathrm{d}_{2}=42 \AA, \mathrm{d}_{3}=18 \AA$ with a magnetic moment per fcc Co atom of $\mu_{\mathrm{Co}}=(1.80 \pm 0.25) \mu_{\mathrm{B}}$ which is in agreement with the calculated ground-state magnetic value [27]. This value is of the order of the bulk value which is $1.64 \mu_{\mathrm{B}}$. First, this example shows that the polarized neutron reflectometry technique provides a direct means to determine the absolute moment per atom within magnetic films. Secondly, it shows that the system $\mathrm{Cu}$ overlayer/18 $\mathrm{Co}$ film/. Cu substrate does not enhance the magnetic moment of $\mathrm{Co}$ in this range of Co thickness.

\section{CONCLUSION}

First, we have outlined the essential background of the theory of the grazing angle polarized neutron rerflectometry. Secondly, we gave some typical examples illustrating the capabilities of the Polarized Neutron Reflectometry to study surface and interface magnetism. From these results, one can conclude that the specular Polarized Neutron Reflectometry provides a non-destructive technique and sensitive to film thicknesses down to a single monolayer. It provides a direct means to determine absolute moment per atom within magnetic films and its orientation relative to the external perturbation. The magnetization profile across the thickness of a bilayer in stratified media could be determined with a resolution of a fraction of a Bohr magneton and a few Angstroms. 


\section{ACKNOWLEDGEMENT}

I am indebted to Pr. J.A.C. Bland from Cambridge University, Dr.L.P. Chernenko from Dubna Research Reactor, Dr. D.A. Korneev from Dubna Research Reactor and Dr. O. Schäerpf from Institut Laüe-Langevin. I owe a great debt to Mrs I. Grange for her invaluable help with the work described here.

\section{REFERENCES}

[1] L.M. Falicov, D.T. Pierce, S.D. Bader, R. Gronsky, K.L.Hathaway, H.J. Hopster, D.N.Lambeth, S.S.P.Parkin, G.Prinz, M. Salamon, I.K. Schuller and R.H. Victora, J. Mat. Res. 5, 1299 (1990).

[2] A. Fert, "Magnetic and Transport properties of Metallic Multilayers.", Material Science Forum, Vol.59 and 60, p.439, (1990).

[3] R. Feder, ed. Polarized Electrons in Surface Physics (World Scientific, Singapore, 1985).

[4] J. Mathon, Rep. Prog. Phys.51 (1988) 1.

[5] G.P. Felcher, Phys. Rev. B24, 1595, (1981).

[6] J. A.C. Bland, D. Pescia and R.F. Willis, Phys. Rev. Lett.58, p1244, (1987).

[7] G.E. Bacon, "Neutron Diffraction.", 3rd edition, Clarendon Press, Oxford, (1975).

[8] J.B. Hatyer, in : "Neutron Diffraction.", edited by H. Dachs, Springer Berlin, p.41, (1978).

[9] J. Penfold and R.K. Thomas, J. Phys. Condens. Matter 2, pp.1369-1412, (1990).

[10] J.Hayter and H.A. Mook, J. Appl. Cryst.22, pp.35-44, (1989).

[11] G. P. Felcher, R. Kleb, D.G. Hinks and W.H. Potter, Phys. Rev.B29, 4843, (1984).

[12] B.Hamelin, Nucl. Instr. and Meth. 135, p.299, (1976).

[13] V. F. Sears,"Neutron Optics.", Clarendon Press. Oxford., (1988).

[14] F. Abelès, Ann. Phys. 5, p.777, (1950).

[15] P. A. Krupchitsky, "Fundamental Research with Polarized Slow Neutrons.", p.3, Springer-Verlag, (1987).

[16] O.Schäerpf, Physica B156-157, pp.631-638, North-Holland, (1989).

[17] F. Mezei and P.A. Dagleish, Comm. Phys.2, pp.41-43, (1977).

[18] A.M. Saxena and B.P. Schoenborn, Acta. Crys. A33, pp. 805-813, (1977).

[19] B. Farnoux, Mat.Res.Soc. Symposium Proceedings, Vol. 166., 95-101,1990.

[20] M.Mâaza, to appear in the Proceeding of International Fermi School on "Industrial Applications of Neutrons." Lerici, Italy, June22-30,(1991).

[21] M.Mâaza, C. Sella, J.P. Ambroise, M. Kâabouchi, M. Milôche, F. Wehling, M. Groos, to be published in J. Phys,(1992).

[22] C.F. Majkrzak and G. Shirane, J. Phys. (Paris),43, C7-215, (1982).

[23] L.P. Chernenko, D.A. Korneev, A.V. Petrenko and N.I. Balalykin, Springer Proceedings in Physics 61"Surface X-ray and Neutron Scattering.", H. Zabel and I.K. Robinson editors, pp.227-231, (1992).

[24] D.A. Korneev, V.V. Pasyuk, A.V. Petrenko and E.B. Dokukin, Springer Proceedings in Physics 61"Surface X-ray and Neutron Scattering.", H. Zabel and I.K. Robinson editors, pp.227-231, (1992).

[25] C.L. Fu, A.J. Freeman and T. Oguchi, Phys. Rev.Lett54, p.2700, (1985).]

[26] H.J. Lauter, J.A.C. Bland, R.D. Bateson and A.D. Johnson, Springer Proceedings in Physics 61"Surface X-ray and Neutron Scattering.", H. Zabel and I.K. Robinson editors, pp.219-222, (1992).

[27] P.M.Marcus and N.L. Moruzzi, Solid. State. Commun. 55, 971, (1985). 